CASE REPORT

Volume 16 Issue 22021

DOI: 10.21315/aos2021.16.2.13

ARTICLE INFO

Submitted: 22/12/2020

Accepted: 10/08/2021

Online: 22/12/2021

\section{Prosthodontic Rehabilitation of Bilateral Maxillary Lateral Incisors Agenesis: A Case Report with One-Year Follow-Up}

\author{
Yew Hin Beh ${ }^{\mathrm{a}, \mathrm{b}}$, Zaihan Ariffin ${ }^{\mathrm{a}^{*}}$ \\ a Prosthodontic Unit, School of Dental Sciences, Universiti Sains \\ Malaysia, 16150 Kubang Kerian, Kelantan, Malaysia \\ ${ }^{b}$ Department of Restorative Dentistry, Faculty of Dentistry, \\ Universiti Kebangsaan Malaysia, 50300 Kuala Lumpur, Malaysia \\ *Corresponding author: zaihan@usm.my
}

To cite this article: Beh YH, Ariffin Z (2021). Prosthodontic rehabilitation of bilateral maxillary lateral incisors agenesis: A case report with one-year follow-up. Arch Orofac Sci, 16(2): $241-251$. https://doi.org/10.21315/aos2021.16.2.13

To link to this article: https://doi.org/10.21315/aos2021.16.2.13

\begin{abstract}
Globally, non-syndromic tooth agenesis is commonly seen in clinical practice. However, its management is often complex and requires a multidisciplinary team approach for the maximal outcome. While various treatment options are possible, considerations for the treatment are not only based on the dentofacial conditions but also cultural and social background and personal preference of the patient. Thus, patientcentred care approach should always be practised for an optimal outcome. In the present case, a patient with established craniofacial growth presenting with bilateral agenesis of maxillary lateral incisors and over-retained deciduous maxillary left canine sought for aesthetic improvements. The patient did not prefer any orthodontic treatment citing a prolonged treatment duration and sub-optimal motivation as a hindrance. Thus, a prosthodontic only approach was taken by providing a conventional cantilever bridge and ceramic veneers to achieve the aims of treatment. This article discusses the possible limitation of such prosthodontic only solution in managing tooth agenesis.
\end{abstract}

Keywords: Aesthetic; cuspid; hypodontia; incisor; tooth agenesis

\section{INTRODUCTION}

Hypodontia is a common dental anomaly defined as the absence of at least one permanent or deciduous tooth germ excluding the third molar (Chung et al., 2008). The tooth germ for the affected tooth or teeth was not developed nor present. Hence, congenitally missing teeth, a term which was commonly used to describe hypodontia is considered a misnomer as congenital means at the point of birth and most teeth in the permanent dentition, which was the most commonly missing set of teeth have not developed at birth. Thus, tooth agenesis is a more appropriate term, reflecting the developmental nature of such anomaly whereby the tooth germ was never developed at any stage of life (Mani et al., 2014). Several other terms were used to describe the pattern of tooth agenesis including hypodontia which has limited up to five missing teeth excluding the third molar, oligodontia when six or more missing teeth and anodontia whereby all permanent tooth germs were absent throughout life (Chung et al., 2008, Mani et al., 2014). 
Tooth agenesis was among the most commonly observed dental developmental anomaly in which its prevalence varies by population and it ranges from $0.3 \%$ to $10.1 \%$ (Mani et al., 2014). It affects more female Caucasians but there is a weak evidence to suggest any gender predilection in the Asian population (Mattheeuws et al., 2004; Mani et al., 2014). Locally, maxillary lateral incisor was the most commonly affected teeth occurred at a prevalence of $1.7 \%$. This was followed by the maxillary and mandibular second premolar and maxillary canines (Mani et al., 2014). Co-existence of other dental anomaly associated with tooth agenesis such as over-retained deciduous teeth, ectopic eruption, microdontia and delayed dental development is common (Garib et al., 2010).

Regardless of its severity, tooth agenesis has an impact on the oral health-related quality of life (OHR-QoL) (Hashem et al., 2013). Such impact warrants an early detection, monitoring, preventive care, intercepting care and providing treatment from childhood. This encompasses the early behavioural management of the patient and their parents, nurturing their motivation and attitude towards dental care which may get more complex in the future (Gill \& Barker, 2015). Active dental treatments that were suggested including general dental preventive care, removable partial dentures, composite restorations and interceptive orthodontic treatments (Gill \& Barker, 2015).

The present case illustrates the management of a patient with non-syndromic tooth agenesis involving both maxillary lateral incisors with an ectopic eruption of both maxillary canines and over-retained deciduous maxillary left canine. The main objective of this article was to highlight the potential drawbacks when only fixed prosthodontic treatments were adopted to manage tooth agenesis.

\section{CASE REPORT}

25-year-old healthy female was referred to the prosthodontic postgraduate clinic due to concerns about the aesthetics of her maxillary anterior teeth that she experienced following enrolment as a dental undergraduate student. She was now keen on improvements after graduated a year ago. She would like to have the anterior spaces closed and to improve the shape of the maxillary canines that had ectopically erupted. She revealed that none of her family members was having tooth agenesis.

It was observed that her facial profile was symmetrical with equal facial proportions and a low smile line (Fig. 1). Other extraoral examinations were unremarkable. An

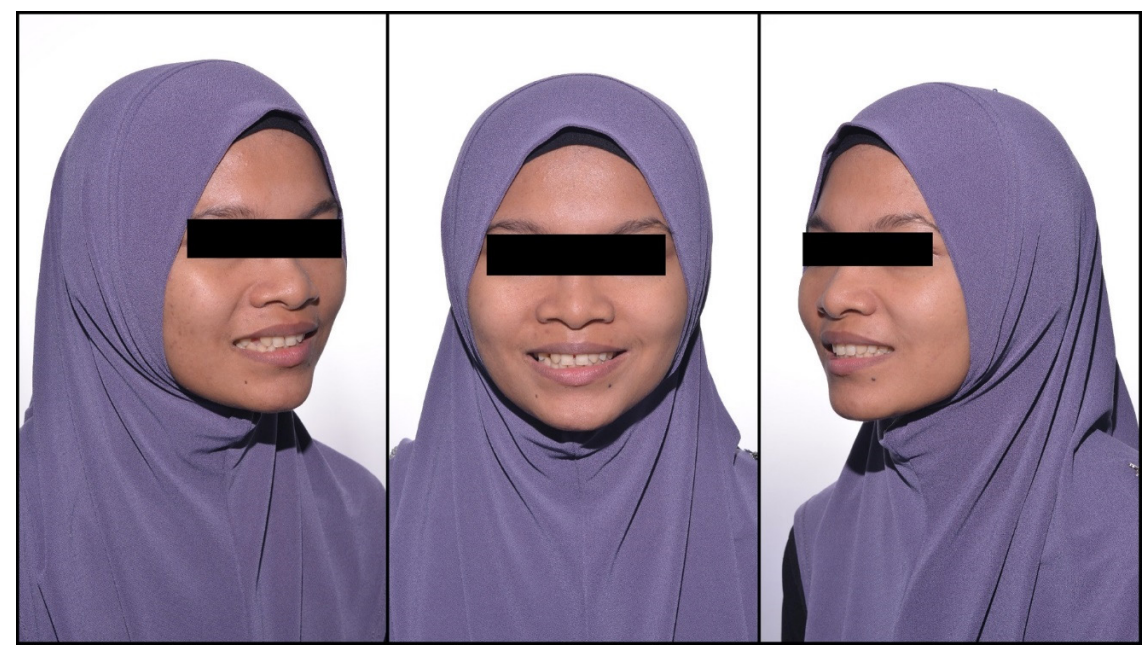

Fig. 1 Pre-treatment extra-oral profile photographs. 
absence of both maxillary lateral incisors with ectopically erupted tooth 13 and 23 was observed in the intra-oral examinations. The maxillary left deciduous canine was over-retained. Diastemas were observed between teeth 11-21 and 13-14 (Fig. 2a2c). Both working sides were in group function involved the contacts from first premolars to the second molars against their
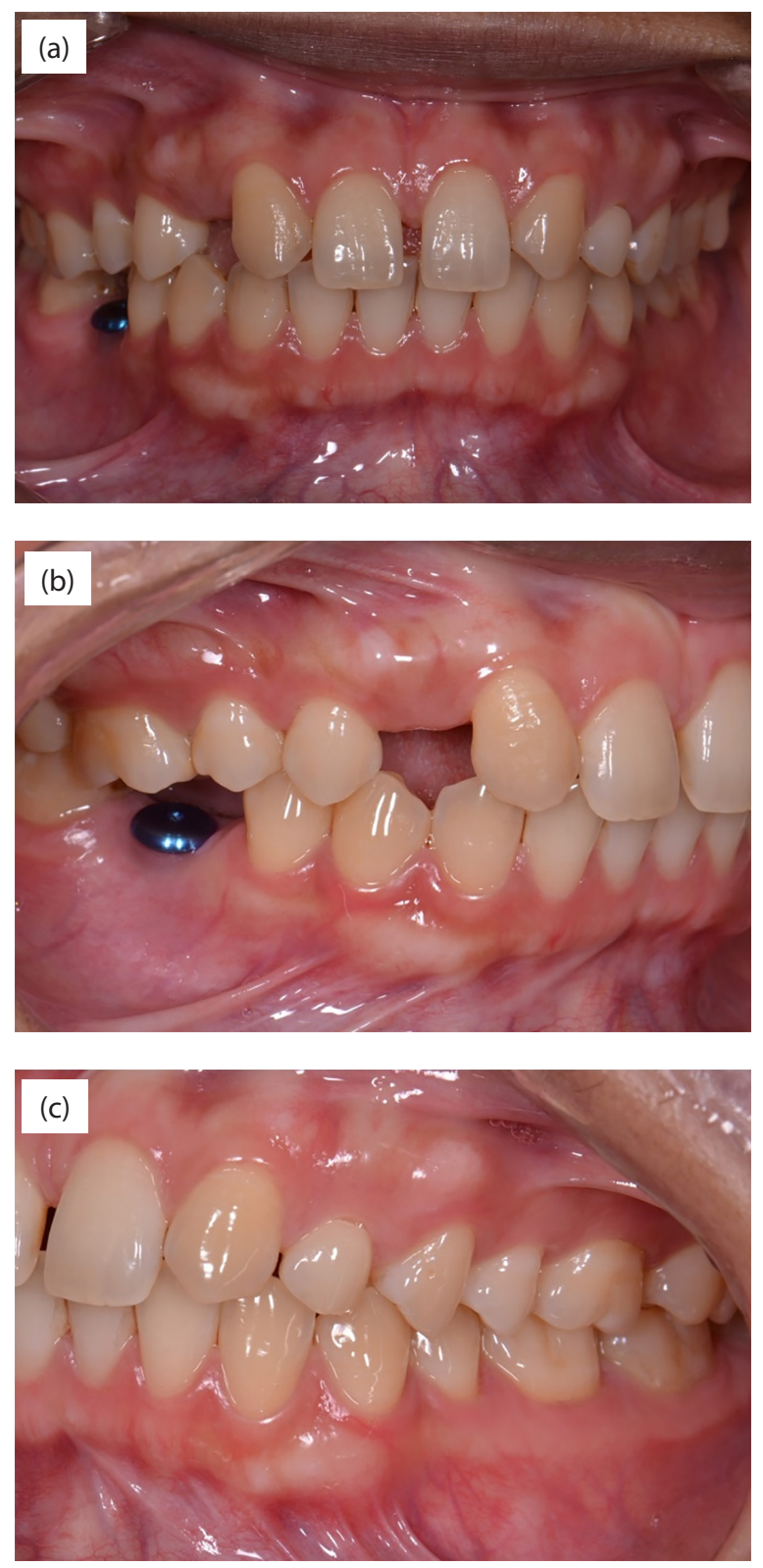

Fig. 2 (a) Pre-treatment intra-oral close-up photo. The healing abutment on 46 implant fixture is not covered in this report as it was managed by another discipline, (b) right buccal view and (c) left buccal view. opposing. There was no occlusal interference upon eccentric mandibular movements. Contacts between maxillary central incisors against all mandibular incisors were evident upon protrusive mandibular movements. Radiographic images in Figs. 3 and 4 showed the absence of both maxillary lateral incisors, malpositioned maxillary canines and overretained maxillary left deciduous canine with minimal root resorption. Treatment options were presented in Table 1, actively discussed for its potential survival rate and complication and was finalised in Table 2 . A conformative approach was planned based on the existing occlusal scheme. The patient was not keen on any orthodontic tooth repositioning to assist in optimal prosthodontic treatments or periodontal surgical intervention to create a harmonious gingival zenith.

After phase 1 treatment, the planned aesthetic changes were discussed based on the diagnostic wax-up (see Fig. 5). Preexisting tooth shade was taken prior to tooth preparation (see Fig. 6). Intra-oral mock-up was done using Protemp 4 (3M, Deutschland $\mathrm{GmbH}$, Germany) followed

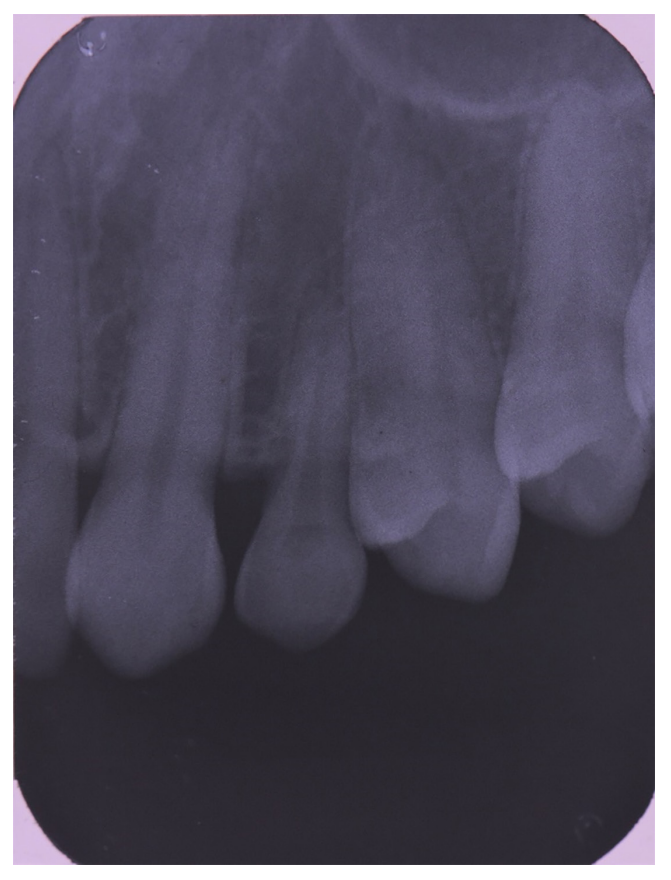

Fig. 3 Periapical radiograph of tooth 63 showing minimal root resorption. 
Table 1 Treatment options proposed and discussed with the patient

\section{Treatment options}

With orthodontic treatment:

1. Extraction of tooth 63 followed by space closure orthodontically. The canine can be left as it is after treatment completion or mimicked to a lateral incisor by ceramic or composite veneer.

2. Extraction of tooth 63 followed by distalisation of tooth 13 and 23 , and diastema closure. The optimal space created for lateral incisors can be restored with:
(a) Implant supported single crown.
(b) Resin bonded bridge (taking into account the occlusal factors post orthodontically in abutment selection).
(c) 3-unit conventional bridge (central incisor and canine as abutment).

Without orthodontic treatment, with extraction of tooth 63:

1. 3-unit conventional bridge on abutment 13 and 14, 23 and 24 with pontic to close the related space. Diastema closure by ceramic or composite veneer.

2. Cantilever bridge on 13 and 23 as abutment and distal pontic. Diastema closure by ceramic or composite veneer.

3. Resin bonded bridge on both canines (13 and 23) as abutment with distal pontic. Diastema closure by ceramic or composite veneer.

Without orthodontic treatment, without extraction of tooth 63:

1. 3-unit conventional bridge on abutment 13 and 14 with pontic to close the related space. Ceramic or composite veneer on 11 and 21 to close the diastema and 23 to mimic the shape onto lateral incisor.

2. Cantilever bridge on 13 as abutment and distal pontic. Ceramic or composite veneer on 11 and 21 to close the diastema and 23 to mimic the shape onto lateral incisor.

3. Resin bonded bridge on 13 abutment with distal pontic. Diastema closure by ceramic or composite veneer. The shape of both canines were to be maintained.

Table 2 Details of the finalised treatment plan

\section{Treatment phase}

Phase 1: Initial phase

Phase 2: Restorative phase

Phase 3: Maintenance phase

\section{Treatment details}

1. Oral hygiene reinforcement.

2. Oral prophylaxis.

1. Conventional cantilever bridge on 13.

(a) Abutment on 13 (shaped to resemble maxillary lateral incisor).

(b) Pontic located distal to 13 (shaped to resemble maxillary premolar).

(c) Material used: porcelain fused to zirconia

2. Veneer on tooth 11,21 and 23.

(a) Veneer 23 shaped to resemble maxillary lateral incisor.

(b) Veneer 11 and 21 with median diastema closure.

(c) Material used: lithium disilicate.

3. Tooth 63 left in-situ without active treatment.

1. Routine review annually.

2. Specific review on the retention of tooth 63 clinically and radiographically.

3. If tooth 63 fail in the future, consideration to prepare tooth 23 as abutment for conventional cantilever bridge and shaped to resemble a maxillary lateral incisor with distal pontic resembling maxillary premolar. 


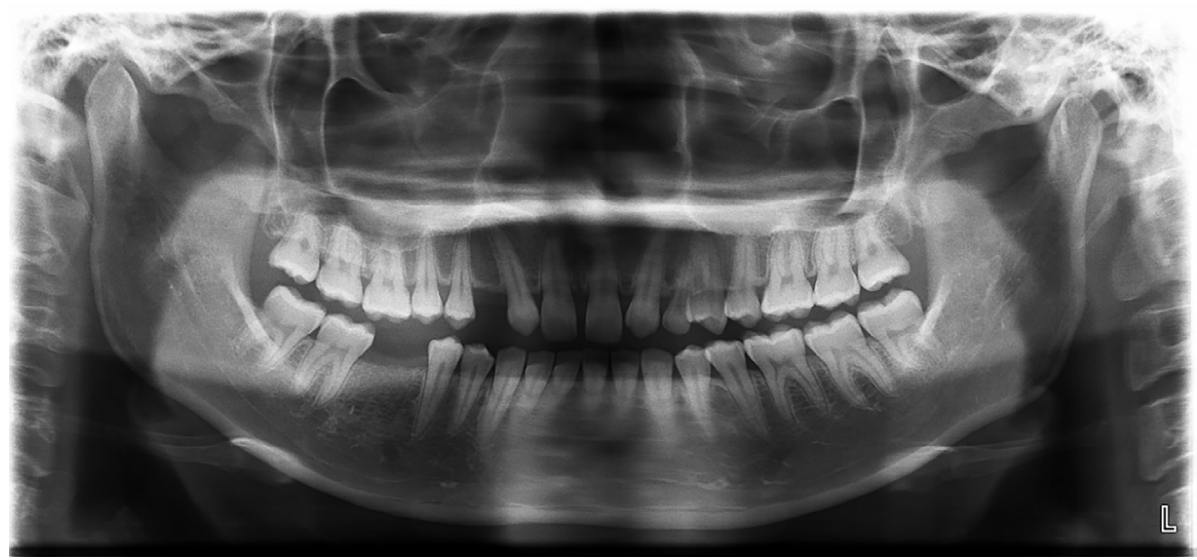

Fig. 4 Pre-treatment orthopantomograph. Note the root angulation of teeth 13 and 23 which was tilted distally corresponding to its usual path of eruption. Agenesis of maxillary lateral incisors resulted in the ectopic positioning of the maxillary canines.

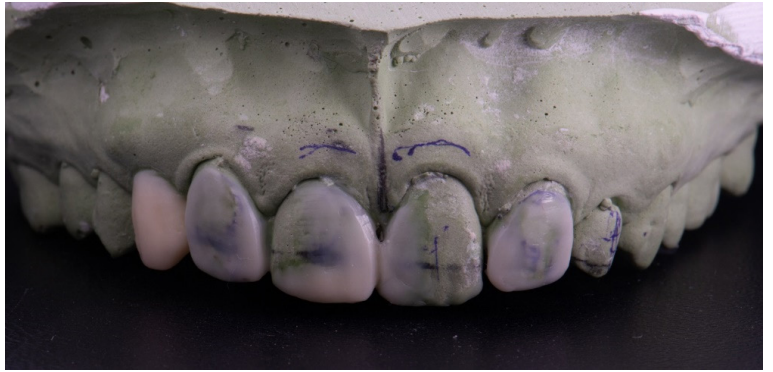

Fig. 5 Diagnostic wax-up made during the planning stage simulating the closure of diastema, tooth shape modification and pontic replacement distal to tooth 13.

by tooth preparation over the mock-up according to the designed fixed prosthesis (Fig. 7). Impression was taken using dualviscosities single-step impression technique with polyvinylsiloxane (Examix, GC America Inc, US). The zirconia bridge was constructed first by an outsourced dental laboratory followed by veneers made by inhouse laboratory.

The finalised prostheses were evaluated for fitting (see Fig. 8). Adjustment on the prostheses was achieved by light pressure, brushing motion using a fine-grit footballshaped diamond bur mounted on a highspeed handpiece with copious water irrigation. All the adjusted surfaces were polished to shine with a silicone ceramic

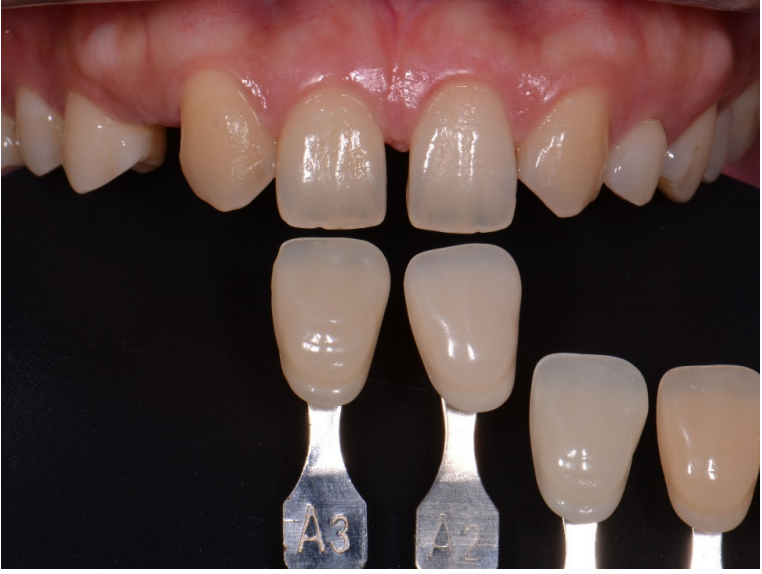

Fig. 6 Teeth shade was determined, and this photo was used to communicate with the ceramist.

polishing kit (Identoflex, Kerr Hawe, Switzerland). The overall aesthetics of the prosthesis was shown to the patient for final agreement before cementation. Porcelain layered zirconia bridge was cemented using Single Bond Universal Adhesive (3M, Deutschland $\mathrm{GmbH}$, Germany) and RelyX Ultimate (3M, Deutschland $\mathrm{GmbH}$, Germany) in translucent shade following surface treatment by air abrasion using $50 \mu \mathrm{m}$ aluminium trioxide particles at $0.2 \mathrm{MPa}$ before cementation. Lithium disilicate (IPS E.max, Ivoclar Vivadent, Liechtenstein) veneers were etched using $9 \%$ hydrofluoric acid (Porcelain etch, Ultradent 

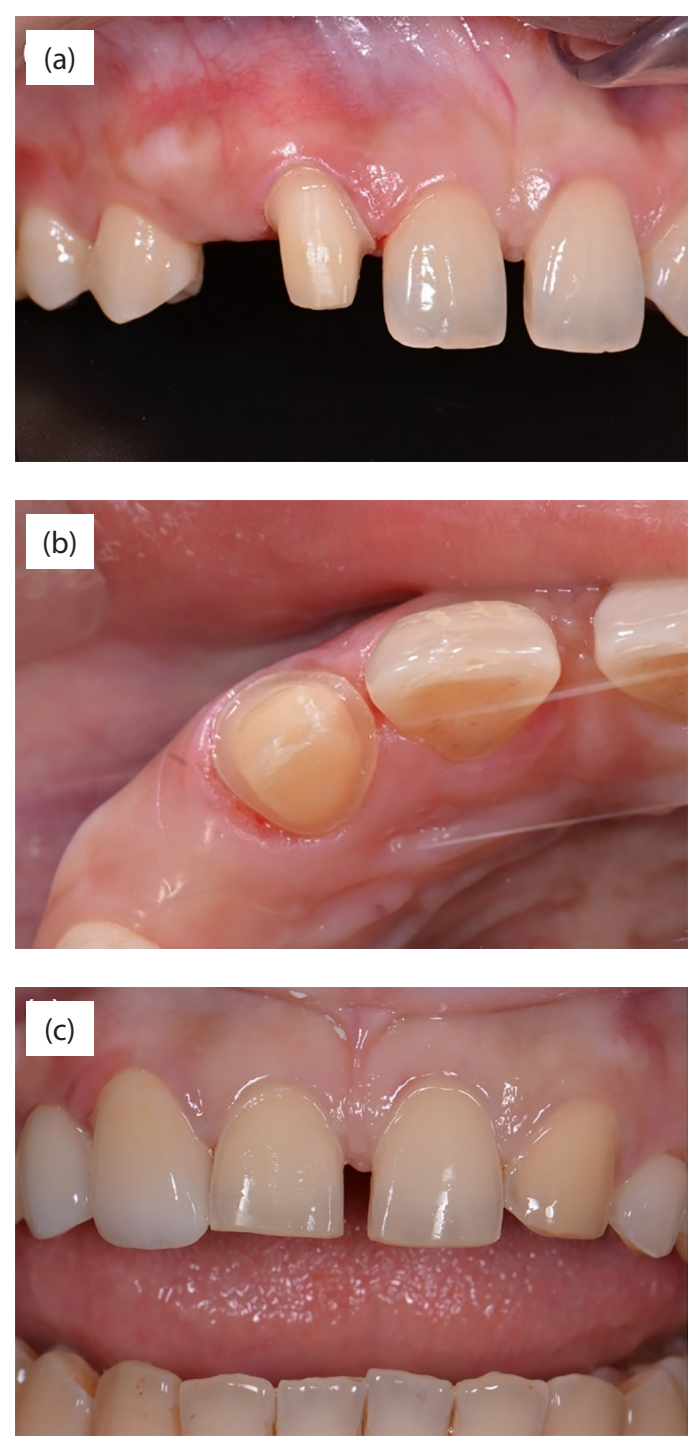

Fig. 7 Teeth preparation, (a) prepared tooth 13,

(b) occlusal view of prepared tooth 13 and

(c) veneer preparation on teeth 11, 21 and 23.

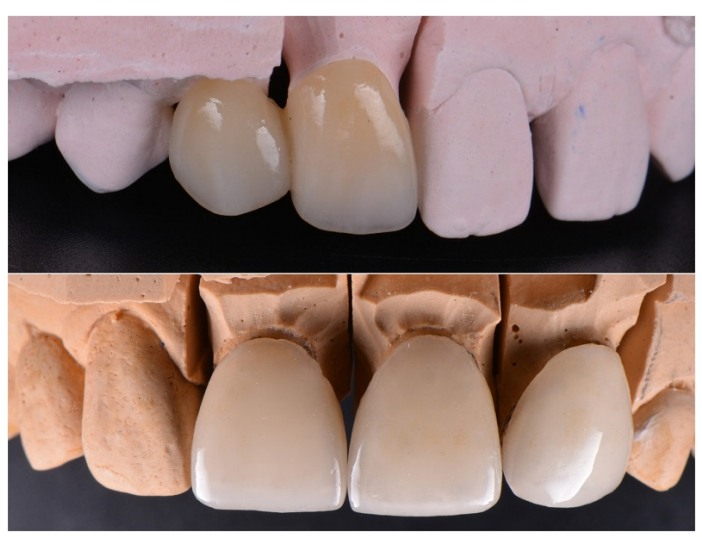

Fig. 8 Completed prostheses positioned onto the master cast for evaluation prior to intra-oral try in.

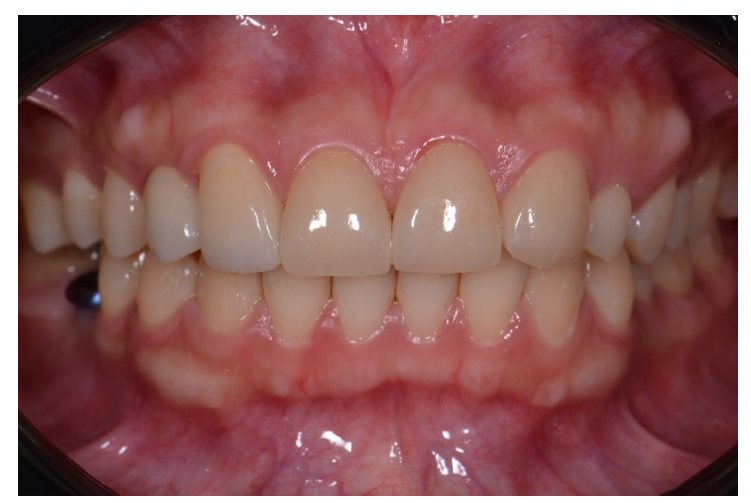

Fig. 9 Close up intra-oral view at one-month review.
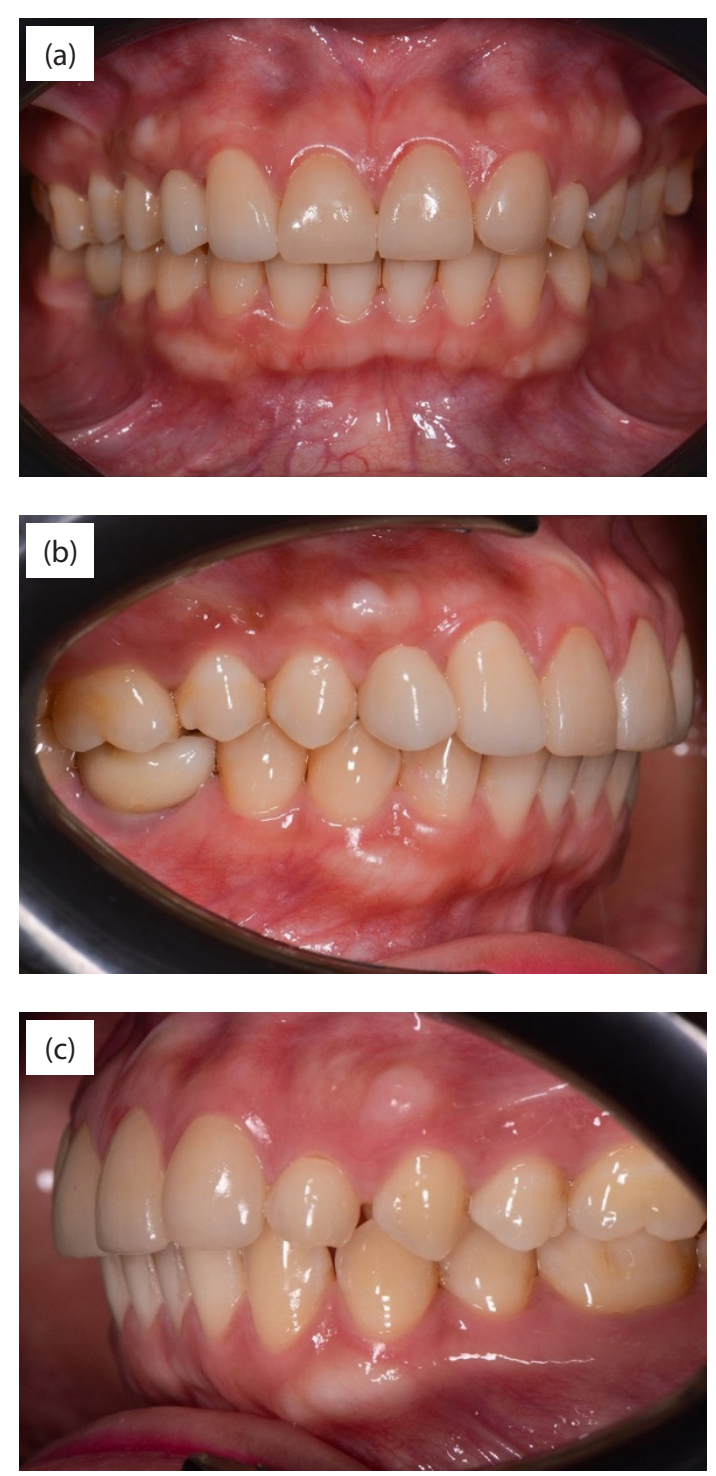

Fig. 10 Close up intra-oral view at one-year review (a) frontal view, (b) right buccal view and (c) left buccal view. 


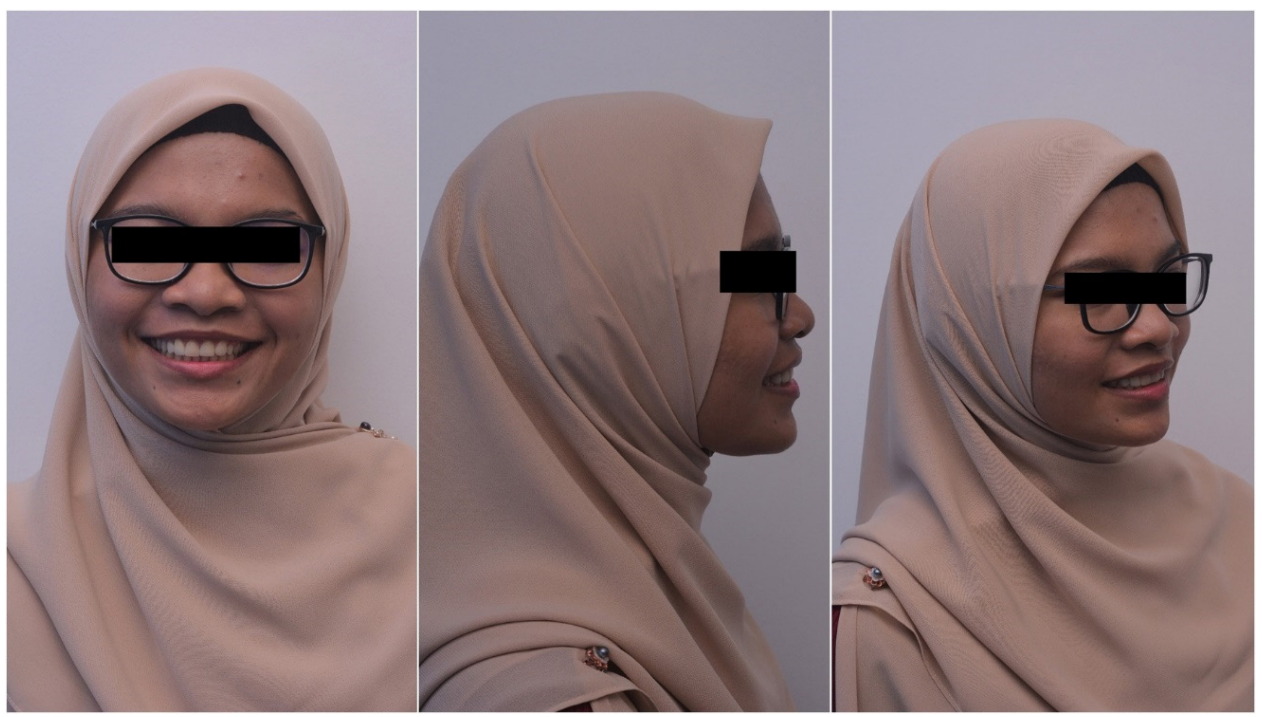

Fig. 11 Extra-oral profile view of the patient at one-year review.

Product Inc, GmbH, Germany) for $90 \mathrm{sec}$ followed by 5 sec 35\% phosphoric acid etch (Ultra-etch, Ultradent Product Inc, US).

A thin layer of silane (Ultradent Product Inc, $\mathrm{GmbH}$, Germany) was applied and allowed air dry before application of Single Bond Universal Adhesive (3M, Deutschland $\mathrm{GmbH}$, Germany) without light curing. The tooth bonding surface was etched with $35 \%$ phosphoric acid etch (Ultra-etch, Ultradent Product Inc, US) for $40 \mathrm{sec}$, washed and dried followed by application of Single Bond Universal Adhesive (3M, Deutschland $\mathrm{GmbH}$, Germany) without light curing. Using RelyX veneer cement (3M, Deutschland $\mathrm{GmbH}$, Germany) translucent shade, veneers were cemented, excess cement was removed and light-cured for $40 \mathrm{sec}$ at each direction. Final polishing using thin extra-fine diamond bur and polishing rubber point.

Upon one-month review, the gingiva was healthy and the interdental papilla between 11 and 21 had filled the interdental space (see Fig. 9). The patient was satisfied with the treatment outcome. The patient's appearance at 1-year recall is presented in Figs. 10 and 11.

\section{DISCUSSION}

Managing patients with tooth agenesis is challenging and requires various treatment options that often involves a multidisciplinary approach (Shafi et al., 2008; Gill \& Barker, 2015; Westgate et al., 2019). This case was presented at an established development of the craniofacial structures in which interceptive treatment was impossible and the treatment goal aimed at the provision of providing a definitive treatment (Gill \& Barker, 2015). The primary concern of the patient was the anterior teeth aesthetics related explicitly to spacing and tooth shape. Hence, she was not interested in treatment from other disciplines, and it eventually became restricted to prosthodontic only options. However, a thorough discussion with the patient was made before agreeing and finalising the treatment plan.

Orthodontic treatment was the treatment of choice in tooth agenesis either to open, close or redistribute the spaces, realignment of tooth roots and enhancing the crown long axis complementing prosthodontic treatment outcome (Shafi et al., 2008; Gill \& Barker, 2015). The existing teeth positions can be redistributed, allowing an optimal space for maxillary lateral incisors which can be replaced with either implant-supported 
single crown, resin-bonded bridges or conventional bridges depending on the final outcome of the orthodontic treatment. Although the space distal to tooth 13 in this case was adequate for reconstruction of a full-sized canine or premolar, an implant supported crown was not possible without prior orthodontic treatment. This issue was evident in the orthopantomograph owing to the root position of tooth 13 which was distally inclined making placement of an appropriately sized implant fixture at an extreme risk for damaging the root of 13 . Alternatively, all the spaces can be closed via orthodontic tooth movements followed by mimicking the maxillary canines to lateral incisors by the provision of ceramic veneers or direct composite veneers. The said approaches complementing the prosthodontic treatment options by means of a better conservation of tooth structure instead of a purely prosthodontic approach as depicted in this case. However, practitioners should be aware that prolonged treatment duration requires good motivation and patient's cooperation in addition to an increased cost (Daniels et al., 2009).

It is possible to maintain a strategically located deciduous teeth, especially when orthodontic treatment was not the treatment of choice (Shafi et al., 2008). The dilemma was related to its prognosis; however, it has been shown that deciduous canine had minimal root resorption over time, so maintaining it was predictable (Haselden et al., 2001). Nevertheless, in the event of potential complication on the retained deciduous tooth, future replacement plans have been made at this point. Thus, in this case, tooth 63 was maintained without any active intervention. The patient agreed to the given option despite the significantly smaller clinical crown of tooth 63 as it was not obvious upon smiling.

The harmonious gingival zenith level for maxillary canine was more apically located and positioned centrally along the tooth long axis (Chu et al., 2009). Some degree of gingival disharmony resulted when maxillary canines were to be camouflaged to resemble maxillary lateral incisors as in this case. This was unfavourable especially for patient with a high smile line, but this patient had a low smile line and the gingival zenith disharmony was not a significant issue. Preventing black triangle during diastema closure required optimal positioning of the contact point. The distance from contact point to interdental bone crest of $5 \mathrm{~mm}$ or less was associated with the presence of interdental papilla (Tarnow et al., 1992). A long contact point may be prescribed to prevent black triangle after median diastema closure.

Veneer is a minimally invasive, predictable treatment option in diastema closure, optimising tooth shape and masking discolouration. Ceramic veneer has an excellent survival of more than $94 \%$ at 10 years (Beier et al., 2012; Aslan et al., 2019). The use of etchable, pressed ceramic for veneer restoration was excellent (Beier et al., 2012; Aslan et al., 2019), however, despite an improved bonding predictability of zirconia material to tooth structure (Blatz et al., 2016), the use of zirconia as veneer was not well documented. There was inadequate evidence to support the role of zirconia veneer in the clinical setting, hence, in this case, it was decided to have a combination of both zirconia bridge and lithium disilicate veneer. Also, based on the manufacturer's instruction, lithium disilicate pressed ceramic was contraindicated as the framework for a cantilever bridge. The preparation of the bulbous canine to receive veneer mimicking a relatively flat labial profile of maxillary lateral incisors puts them at risk for dentinal exposure at the most bulbous aspect of the tooth. By additive wax-up and preparing depth guide over the mock-up material, the risk for dentinal exposure was reduced and the restoration survival was more predictable (Burke, 2012). Bonding of glass ceramic to tooth structure was well described previously whereby the surface treatment by using hydrofluoric etching and silane application on the glass ceramic followed by resin cement produced a clinically optimal bonding (Blatz et al., 2003). 
Cantilever fixed dental prosthesis (FDP), comparatively more conservative than conventional FDP as only one abutment was involved. With $91.4 \%$ survival estimates after five years was slightly lower compared to conventional FDP design which had a fiveyear survival of $93.8 \%$ but at the expense of reduced biological cost (Pjetursson et al., 2007). Cantilever FDP allowed tooth shape modification in contrast to resinbonded bridge; hence this option was chosen. Loss of abutment tooth vitality was the main issue with full coverage tooth preparation with $5.4 \%$ reported incidence in five years for a cantilever FDP yet still lower than conventional FDP which stand at $6.1 \%$ (Pjetursson et al., 2007). The distal cantilever was often associated with an increased risk for mechanical complications due to the increased levering biomechanical forces. However, in this patient, mesial cantilever bridge connected to abutment tooth 14 was more invasive compared to utilising abutment tooth 13 as tooth 13 required shape modification. Hence, careful design of the pontic was essential; reduced buccolingual dimension, reduced crown height along with careful occlusal scheme design whereby there were light contact on the pontic at the intercuspal position and no contacts at lateral excursions. Proper bonding of zirconia-based prosthesis ensured adequate and improved prosthesis retention, especially in a high demand situation. Previously, zirconia-based prosthesis was associated with a significantly higher incidence of loss of retention. Special consideration was given in this case to bond the zirconia-based cantilever bridge to the prepared abutment using resin cement. Due to the minimal to no silica component in the zirconia structure, hydrofluoric etch was not possible. Air abrasion was done to create micromechanical retentive intaglio surface followed by utilising a 10-methacryloyloxydecyl-dihydrogen phosphate (10-MDP) containing universal bonding system and resin cement to bond the prosthesis. This bonding protocol was proven to enhance zirconia bonding to tooth structure resulting in a predictable prosthesis retention (Blatz et al., 2016; dos Santos et al., 2019).

One major limitation in this case was the patient's refusal towards orthodontic treatment. If a multi-disciplinary management was to be accepted by the patient, the overall treatment outcome can be more conservative to the dental tissue with the provision of implant restoration and minimally invasive ceramic veneers. The other drawbacks in this case were the sub-optimal gingival harmony, the possible biomechanical disadvantage for a distal facing cantilever bridge, potential biological complications from the extensive tooth preparation for full coverage restoration, the challenge to create a harmonious teeth shade due to the dissimilar materials utilised and the predictability to retain the deciduous tooth. Nevertheless, each drawback was managed adequately to reduce the occurrence of potential complications. Considerations between aesthetic demands and conservation of tooth structure shall be balanced and this case served as a guide for practitioners when encountering cases of similar nature. A more conservative, minimally invasive treatments shall always be emphasised adequately before embarking on invasive treatment options.

\section{CONCLUSION}

Ideally, for the maximal outcome, tooth agenesis requires a multidisciplinary care. However, when this is not feasible due to various limiting factors, understanding the possible limitations in term of tissue conservation, aesthetic outcome, biomechanical considerations of the related prosthesis and the possible risks of complications, and addressing the limitations to the patient is necessary for to achieve an optimal result, thereby ensuring an individualised, patient-centred treatment approach. 


\section{ACKNOWLEDGEMENTS}

The authors clarify that all the appropriate consent was taken to the disclosure of clinical information and photographs in this article for publication. The patient acknowledges that she remained unnamed in this report but there is no guarantee of full anonymity. We wish to acknowledge Research University Grant (1001/PPSG/8012282) which granted to the corresponding author by the Universiti Sains Malaysia in supporting the patient's management and the work in this article.

\section{REFERENCES}

Aslan YU, Uludamar A, Özkan Y (2019). Clinical performance of pressable glassceramic veneers after 5, 10, 15 and 20 years: A retrospective case series study. $\mathcal{F}$ Esthet Restor Dent, 31(5): 415-422. https://doi.org/10.1111/jerd.12496

Beier US, Kapferer I, Burtscher D, Dumfahrt H (2012). Clinical performance of porcelain laminate veneers for up to 20 years. Int $\mathcal{F}$ Prosthodont, 25(1): 79-85.

Blatz MB, Alvarez M, Sawyer K, Brindis M (2016). How to bond zirconia: The APC concept. Compend Contin Educ Dent, 37(9): 611-618.

Blatz MB, Sadan A, Kern M (2003). Resinceramic bonding: A review of the literature. F Prosthet Dent, 89(3): 268-274. https://doi.org/10.1067/mpr.2003.50

Burke FJT (2012). Survival rates for porcelain laminate veneers with special reference to the effect of preparation in dentin: A literature review. F Esthet Restor Dent, 24(4): 257-265. https://doi.org/10.1111/j.17088240.2012.00517.x

Chu SJ, Tan JH, Stappert CF, Tarnow DP (2009). Gingival zenith positions and levels of the maxillary anterior dentition. $\mathcal{F}$ Esthet Restor Dent, 21(2): 113-120. https://doi.org/ 10.1111/j.1708-8240.2009.00242.x
Chung CJ, Han JH, Kim KH (2008). The pattern and prevalence of hypodontia in Koreans. Oral Dis, 14(7): 620-625. https://doi.org/10.1111/j.1601-0825.2007 $.01434 . \mathrm{x}$

Daniels AS, Seacat JD, Inglehart MR (2009). Orthodontic treatment motivation and cooperation: A cross-sectional analysis of adolescent patients' and parents' responses. Am f Orthod Dentofacial Orthop, 136(6): 780-787. https://doi.org/10.1016/ j.ajodo.2007.11.031

dos Santos RA, de Lima EA, Mendonça LS, de Oliveira JE, Rizuto AV, de Araújo Silva Tavares ÁF et al. (2019). Can universal adhesive systems bond to zirconia? $\mathcal{F}$ Esthet Restor Dent, 31(6): 589-594. https://doi .org $/ 10.1111 /$ jerd.12521

Garib DG, Alencar BM, Lauris JRP, Baccetti $T$ (2010). Agenesis of maxillary lateral incisors and associated dental anomalies. Am $\mathcal{f}$ Orthod Dentofacial Orthop, 137(6): 732.e1-732.e6. https://doi.org/10.1016/ j.ajodo.2009.12.024

Gill DS, Barker CS (2015). The multidisciplinary management of hypodontia: A team approach. Br Dent f, 218(3): 143-149. https://doi.org/10.1038/sj.bdj.2015.52

Haselden K, Hobkirk JA, Goodman JR, Jones SP, Hemmings KW (2001). Root resorption in retained deciduous canine and molar teeth without permanent successors in patients with severe hypodontia. Int $\mathcal{F}$ Paediatr Dent, 11(3): 171-178. https://doi.org/10.1046/j $.1365-263 x .2001 .00257 . x$

Hashem A, Kelly A, O'Connell B, O'Sullivan $M$ (2013). Impact of moderate and severe hypodontia and amelogenesis imperfecta on quality of life and self-esteem of adult patients. F Dent, 41(8): 689-694. https://doi.org/10.1016/j.jdent.2013.06.004

Mani SA, Mohsin WSY, John J (2014). Prevalence and patterns of tooth agenesis among Malay children. Southeast Asian $\mathcal{F}$ Trop Med Public Health, 45(2): 490-498. 
Mattheeuws N, Dermaut L, Martens G (2004). Has hypodontia increased in Caucasians during the 20th century? A meta-analysis. Eur $\mathcal{F}$ Orthod, 26(1): 99-103. https://doi .org/10.1093/ejo/26.1.99

Pjetursson BE, Brägger U, Lang NP, Zwahlen $M$ (2007). Comparison of survival and complication rates of tooth-supported fixed dental prostheses (FDPs) and implantsupported FDPs and single crowns (SCs). Clin Oral Implants Res, 18(Suppl 3): 97-113. https://doi.org/10.1111/j.16000501.2007.01439.x

Shafi I, Phillips JM, Dawson MP, Broad RD, Hosey MT (2008). A study of patients attending a multidisciplinary hypodontia clinic over a five year period. $\mathrm{Br}$ Dent $\mathcal{F}$, 205(12): 649-652. https://doi.org/10.1038/ sj.bdj.2008.1080
Tarnow DP, Magner AW, Fletcher P (1992). The effect of the distance from the contact point to the crest of bone on the presence or absence of the interproximal dental papilla. I Periodontol, 63(12): 995-996. https://doi.org/10.1902/jop.1992.63.12.995

Westgate E, Waring D, Malik O, Darcey J (2019). Management of missing maxillary lateral incisors in general practice: Space opening versus space closure. $\mathrm{Br}$ Dent $\mathcal{F}$, 226(6): 400-406. https://doi.org/10.1038/ s41415-019-0082-4 